\title{
Nuevos registros de gasterópodos para el mar tropical del Perú
}

\author{
New records of gastropods from the tropical sea of Peru
}

\author{
Carlos Paredes ${ }^{1,2}$, Katherine Altamirano1, Leonardo Romero ${ }^{1}$ y Franz Cardoso ${ }^{1,2}$
}

1 Laboratorio de Biología y Sistemática de Invertebrados Marinos, Facultad de Ciencias Biológicas, Universidad Nacional Mayor de San Marcos, Apdo. 11-0058, Lima 11, Perú. E-mail Carlos Paredes: cparedesq@unmsm.edu.pe

2 Departamento de Malacología, Museo de Historia Natural, Universidad Nacional Mayor de San Marcos, Apdo. 14-0434, Lima 14, Perú.

Presentado: $19 / 11 / 2008$ Aceptado: $15 / 12 / 2008$ Publicado online: $26 / 02 / 2009$

\section{Resumen}

Cinco especies de gasterópodos: Northia pristis (Deshayes in Lamarck, 1844), Macron aethiops (Reeve, 1847), Latirus concentricus (Reeve, 1847), Terebra elata, Hinds, 1844 y Carinodrilla adonis Pilsbry \& Lowe, 1932, se reportan por primera vez para aguas tropicales del mar peruano. Se da información sobre la distribución, el hábitat y comentarios relevantes sobre cada una de las especies.

Palabras claves: Mollusca, Gastropoda, Caenogastropoda, nuevos registros, Perú.

\section{Abstract}

The following species of Gastropoda have been recorded for the first time for tropical Peruvian waters: Northia pristis (Deshayes in Lamarck, 1844), Macron aethiops, (Reeve, 1847), Latirus concentricus (Reeve, 1847) Terebra elata Hinds, 1844, y Carinodrilla adonis Pilsbry \& Lowe, 1932. Information on distribution geographical and same relations ecological is presented.

Keywords: Mollusks, Gastropoda, Caenogastropoda, new records, Peru.

\section{Introducción}

En el Perú, país signatario del Convenio sobre Diversidad Biológica (1992), son muy escasos los estudios sobre la malacofauna marina, principalmente por no existir una política de apoyo a la investigación.

Nosotros desde hace varios años venimos trabajando en el inventario y la determinación taxonómica actualizada de los moluscos marinos (Paredes et al. 1998, 1999; Ramírez et al. 2003; Paredes et al. 2005, Paredes y Cardoso, 1998, 2001a, 2001b, 2003, 2007, 2008).

En esta oportunidad, dentro del estudio de los gasterópodos de la subclase Caenogastropoda, que viven en los niveles sublitorales o infralitorales del mar en cualquier tipo de fondo, incluyendo aquellas especies que siendo sublitorales alcanzan la zona intermareal, se da a conocer el hallazgo de cinco especies de gasterópodos que se registran por primera vez para nuestro mar tropical.

\section{Material y métodos}

La colecta del material biológico se realizó mediante dragado o buceo autónomo en el sublitoral durante los trabajos de campo programados dentro de una expedición organizada por el Instituto del Mar del Perú, realizada en el mes de abril del año 2007, en la cual participó la bióloga Katherine Altamirano.

El material fue fijado en formol al 7\% neutralizado con bórax, y conservado en alcohol etílico al 70\%. La determinación taxonómica se realizó utilizando la bibliografía especializada. Para el ordenamiento sistemático se siguió a Skoglund (2002). El material está depositado en las colecciones del Museo de Historia Natural de la Universidad Nacional Mayor de San Marcos (MUSM) y el Laboratorio de Biología y Sistemática de Invertebrados Marinos de la Facultad de Ciencias Biológicas de la Universidad Nacional Mayor de San Marcos (LaBSIM).

\section{Resultados y discusión \\ Subclase Caenogastropoda \\ Orden Neotaenioglosa \\ Suborden Neogastropoda}

Superfamilia MURICOIDEA

\section{Familia Buccinidae Rafinesque, 1815 \\ Subfamilia Photinae \\ Genero Northia Gray, 1847}

Northia pristis (Deshayes in Lamarck, 1844)

Figura 1

Northia pristis (Deshayes in Lamarck, 1844); Gray, 1857: 18, pl. 84a, fig, 4; Keen, 1971: 568, fig. 1137; Skoglund, 2002: 127.

Concha espiral típica, sólida y pesada; la vuelta corporal es alta y su altura excede la mitad de la altura total; espira alta y acicular con hasta 7 vueltas; la abertura presenta fuertes espinas en el borde sobresaliente del labio externo, las que se proyectan internamente como estrías; callo parietal estrecho y hay un diente curvo en el extremo distal del labio columelar; una foseta sifonal rodea el corto canal sifonal y el fasciolo está bien desarrollado; la vuelta corporal es tabulada bajo la sutura y con la última vuelta de la espira son lisas; las vueltas restantes de la espira presentan costillas axiales y cuerdas espirales, este tipo de escultura es progresivamente más fuerte hacia el apex. Coloración parda claro olivácea, interior de la abertura blanquecino. Longitud, $63,0 \mathrm{~mm}$.

Distribución: Mazatlán, México a Ecuador (Keen, 1971).

Hábitat: Fondo areno fangoso sublitoral, entre 60 y 350 metros de profundidad (Alamo y Valdivieso, 1987). Sólo se colectaron conchillas en la playa arenosa, generalmente ocupadas por cangrejos ermitańos.

Nueva localidad: Tumbes (Puerto Pizarro).

Material examinado: 2 lotes, 6 ejemplares (LaBSIM) (MUSM). Colectores: V. Rivadeneira, 14.06.1991; C. Paredes, 02.10.2005.

Observaciones: En Puerto Pizarro se puede encontrar conchillas de esta especie en la playa arenosa durante la bajamar y cerca de las embarcaciones de los pescadores. Posiblemente se trate de material arrojado por ellos después de sus faenas de pesca. Es conocido como "caracol madera" por la coloración y textura de la conchilla. 


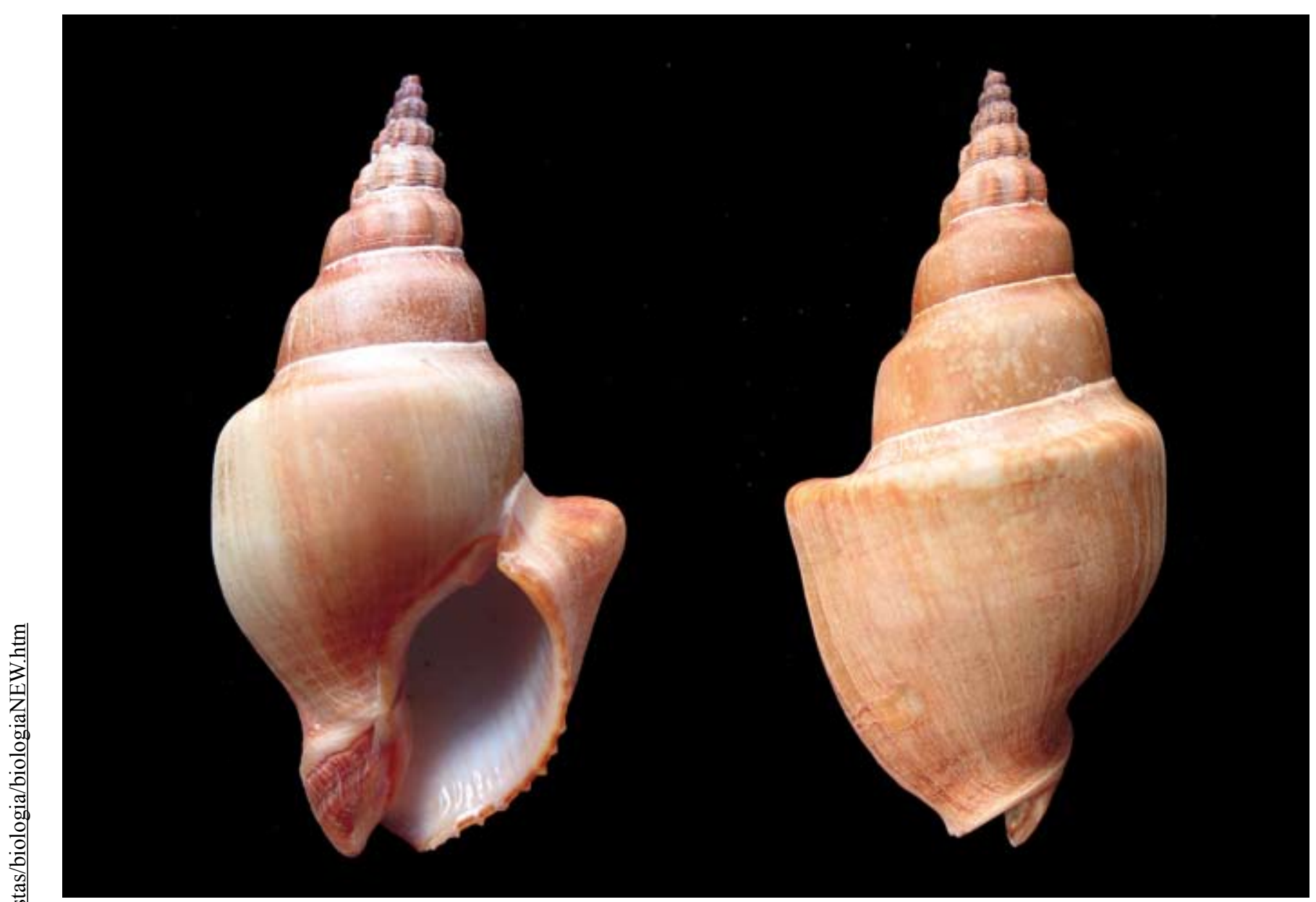

Figura 1. Northia pristis, longitud $63,0 \mathrm{~mm}$.
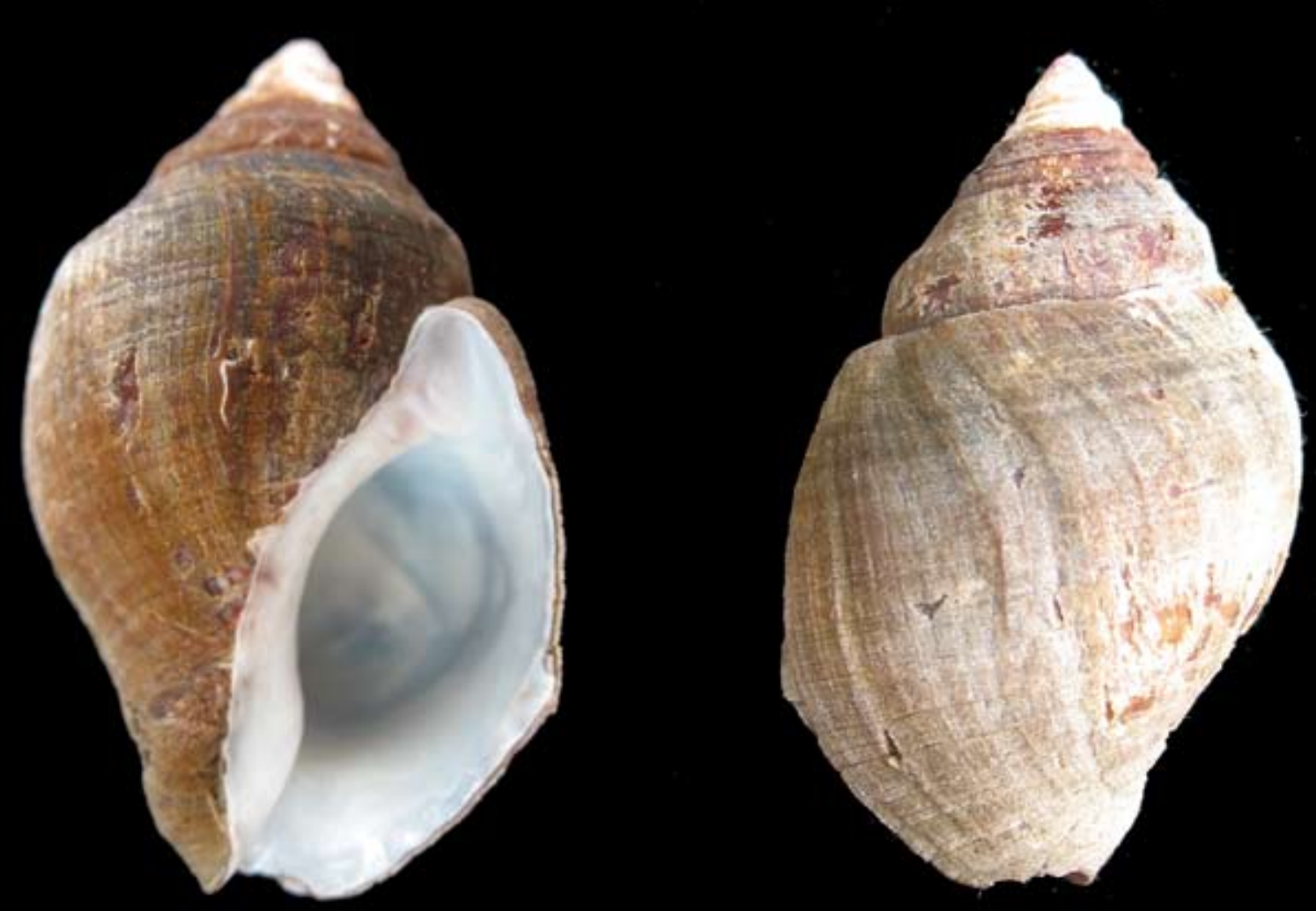

Figura 2. Macron aethiops, longitud 46,0 mm. 
Familia Pseudolividae Crossmann, 1991

Genero Macron H. \& A. Adams, 1853

\section{Macron aethiops (Reeve, 1847)}

Figura 2

Buccinum aethiops Reeve, 1847

Macron kellettii A. Adams, 1854

Macron orcutti Dall, 1918; Keen, 1971: 566, fig. 1132; Abbott, 1974: 221

Macron aethiops, Keen, 1971: 566, fig. 1131; Abbott, 1974: 21, fig. 2430

Concha oval, sólida, con espira corta, apex agudo y periostraco adherente de color verde muy oscuro; abertura en forma de boca, labio externo crenulado en su mitad distal, labio interno con tres pliegues posteriores suaves, columela cóncava, callo columelar estrecho y fasciolo desarrollado, canal sifonal muy corto; superficie de la concha casi lisa, sólo con incisiones espirales que son más evidentes en la base y en la espira. El color externo de la concha es blanco y el interior de la abertura blanco con una tonalidad purpúrea. Longitud, $46,0 \mathrm{~mm}$.

Distribución: Playa San Ramón (30 45’ N) hasta 10 millas al norte de Cabo San Lucas, Baja California Sur, México (DuShane, 1989); y norte del Golfo de California, México hasta la Isla Danzante (Mulliner, 1996).

Hábitat: Sublitoral, en fondos arenofangosos.

Nueva localidad: Piura (Bahía de Sechura, Bayovar).

Material examinado: 1 lote, 1 ejemplar (LaBSIM); colector: M. Apaza y L. Romero; fecha: 19.08.2006
Observaciones: Keen (1971) indica que hay una completa gradación en la ornamentación de la conchilla, que es fuertemente acostillada en $\boldsymbol{M}$. aethiops, y casi lisa, sólo con una costilla basal en $\boldsymbol{M}$. kellettii. Keen, op. cit., refiere que E. A. Smith (1903), revisó los tipos de estas especies y concluyó que se trataba de una sola especie. Asimismo, Keen deja pendiente confirmar si $\boldsymbol{M}$. orcutti solo es una forma más pequeña y lisa de M. aethiops. Este problema fue resuelto por Abbott (1974) y no conocemos de cambios posteriores. Esta especie es un importante depredador de ostras juveniles (Crassostrea gigas). En la Bahía de San Quintín (Baja California, México), puede consumir hasta 4 juveniles por día, causando una importante pérdida económica a los marisqueros artesanales (Rodríguez, 2008).

\section{Familia FasciolariIdae}

Subfamilia Peristerninae Bullock, 1974

Género Latirus Montfort, 1810

\section{Latirus concentricus (Reeve, 1847)}

$$
\text { Figura } 3
$$

Latirus concentricus Reeve, 1847. Con. Icon., Turbinilla, figs. 2, 44; Dall, 1909: 212.; Keen, 1971: 613, fig. 1328; Skoglund, 2002: 142

Concha fusiforme, sólida, con el canal sifonal y la espira de, aproximadamente, igual longitud; labio externo crenulado y con dentículos internos, labio columelar blanco anaranjado con tres pliegues; escultura presenta costillas axiales sobre las que se levantan nódulos; el periostraco es pardo oscuro y adherente. Color externo de la concha blanco anaranjado, interior de la abertura blanco violáceo. Longitud, 44,8 $\mathrm{mm}$.

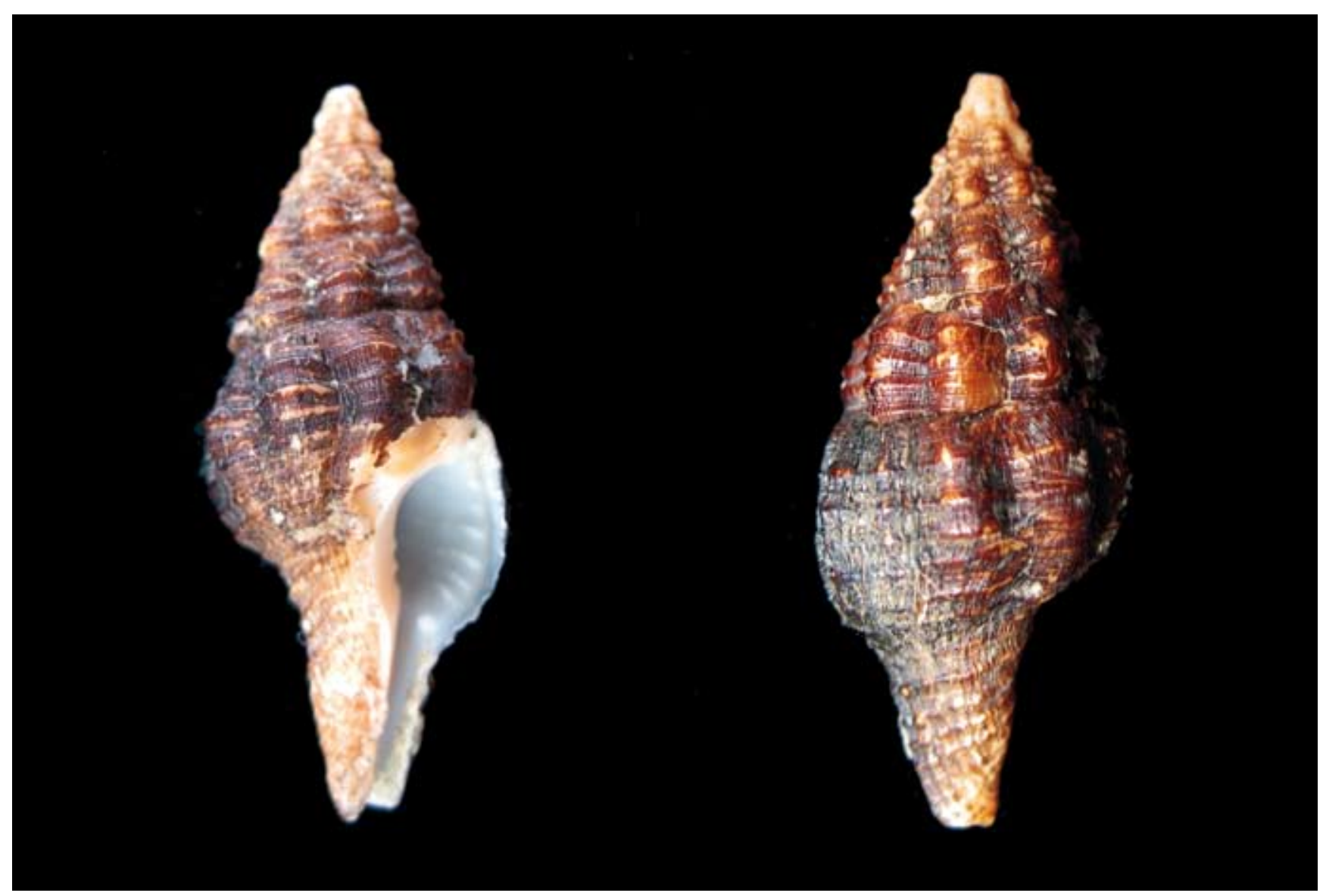

Figura 3. Latirus concentricus, longitud $44,8 \mathrm{~mm}$. 


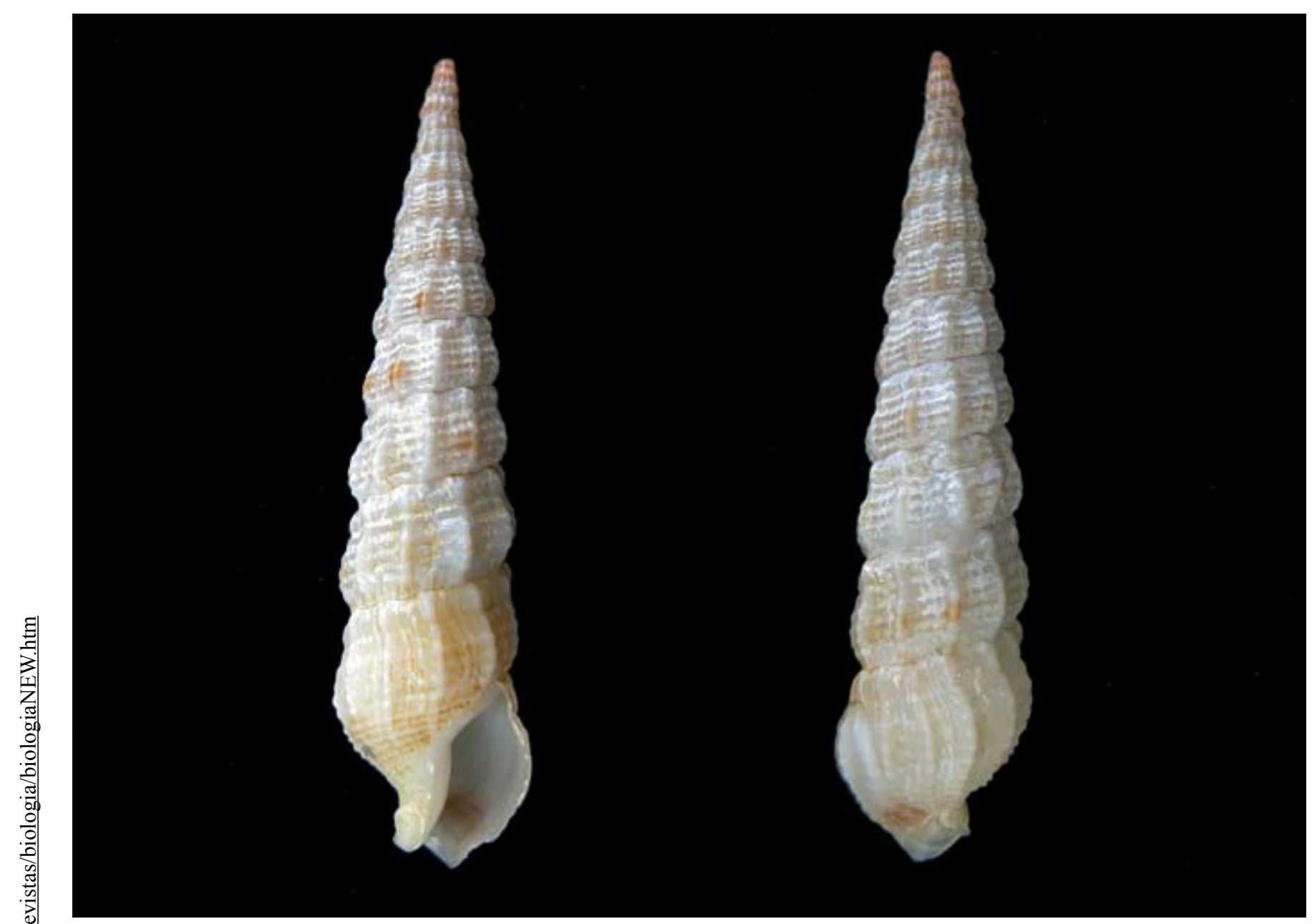

Figura 4. Terebra elata, longitud $44,4 \mathrm{~mm}$.
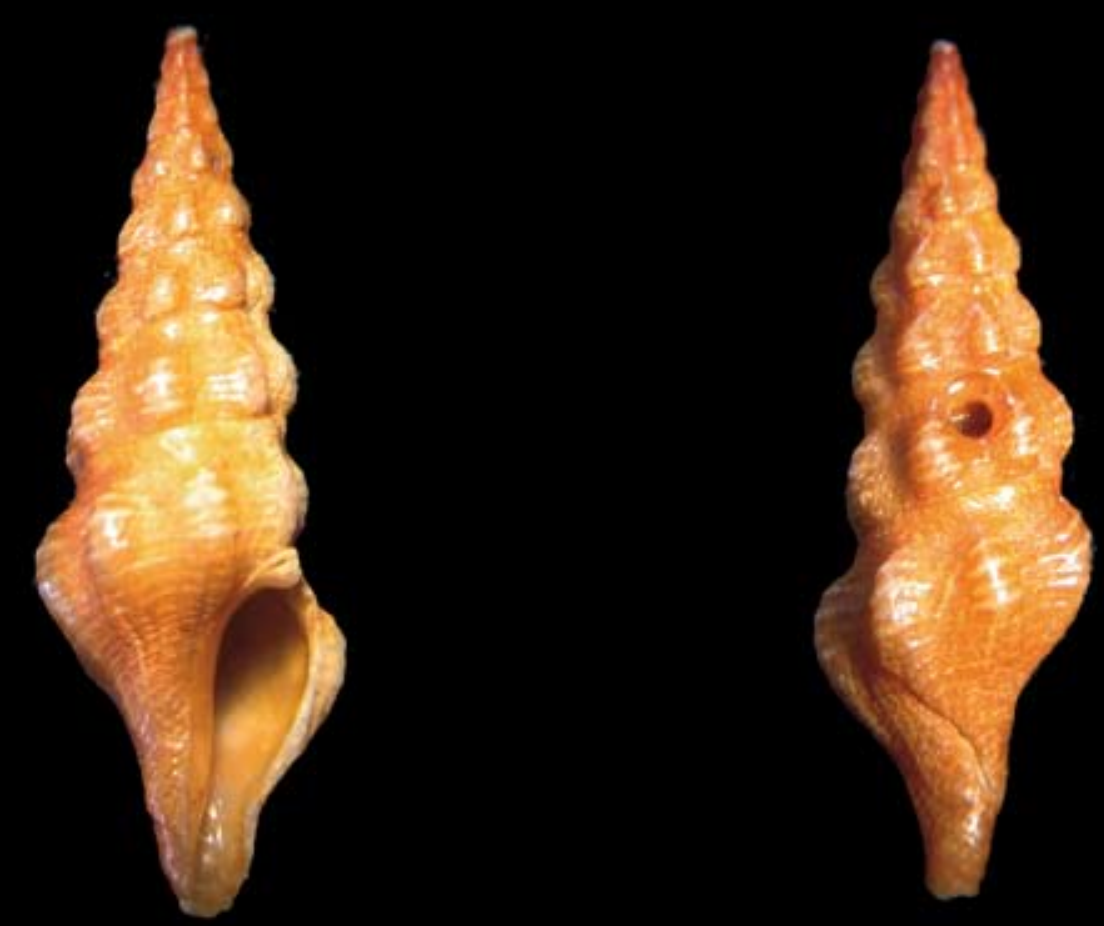

Figura 5. Carinodrilla adonis, longitud 21,7 mm. 
Distribución: Guaymas, México a Ecuador y posiblemente, Panamá (Keen, 1971).

Hábitat: Sublitoral arenoso, a 13,5 m de profundidad.

Nuevas localidades: Tumbes (Punta Sal Chica), Piura (Ensenada Peña Mala, Peńa Parada).

Material examinado: 3 lotes, 4 ejemplares (LaBSIM) (MUSM). Colector K. Altamirano; Fecha: 16-18.04.2007

\section{Suborden MuricoideI}

Superfamilia Conoidea

Familia Terebridae H. \& A. Adams, 1854

Género Terebra Bruguiere, 1789

Terebra elata Hinds, 1844

\section{Figura 4}

Terebra elata Hinds, 1844: Proc. Zool. Soc. London, pp.149-59; Keen, 1971: 676, fig. 1535; Skoglund, 2002: 166.

Conchilla con la forma típica del género, con el canal sifonal algo dirigido hacia la izquierda; borde del labio externo ligeramente crenulado, labio columelar algo cóncavo y sin pliegues; una banda estrecha rodea el canal sifonal y el fasciolo está desarrollado; escultura presenta costillas axiales en todas las vueltas, las que están cruzadas por cuerdas espirales, y una banda subsutural con nódulos alargados situados en la dirección de las costillas. Color exterior blanquecino con algunas manchas pardas, interior de la abertura blanco brillante deja traslucir las manchas pardas externas. Longitud, 44,4 $\mathrm{mm}$.

Distribución: Sur de México a Ecuador; Isla de Coco, Costa Rica (Skoglund, 2002).

Hábitat: Infralitoral areno-fangoso. Colectado hasta 77 metros de profundidad (Hendrickx y Toledano, 1994).

Nueva Localidad: Tumbes (El Arco).

Material examinado: 1 lote, 2 ejemplares LaBSIM). Colector: K. Altamirano; Fecha: 22.10.2006

\section{Familia Turridae Swainson, 1840 \\ Subfamilia Crassispirinae \\ Genero Carinodrilla Dall, 1919}

\section{Carinodrilla adonis Pilsbry \& Lowe, 1932}

\section{Figura 5}

Carinodrilla adonis Pilsbry, 1932: Proc. Acad. Nat. Sci. Philadelphia vol. 84, pp.:33-144, 7 figs., 1-17 pls., 2 photographs; Keen, 1971: 728, fig. 1721; Skoglund, 2002: 175.

Conchilla fusiforme con la vuelta corporal aproximadamente de igual longitud que la espira; la periferia es redondeada y el canal sifonal es relativamente corto; seno profundo con sus paredes dirigidas hacia el ápice; callo columelar bien desarrollado alcanza la entrada del seno; escultura presenta costillas axiales prominentes cruzadas por cuerdas espirales notorias en la vuelta corporal y la última vuelta de la espira, luego las cuerdas son débiles y desaparecen en las primeras vueltas. La coloración es pardo canela. Longitud, 21,7 mm.
Distribución: Frente a Roca Consag, Golfo de California, México (DuShane \& Brennan, 1969), al sur hasta Bahía Santa Elena, Ecuador y las Islas Galápagos (Keen, 1971).

Hábitat: Sublitoral, fondo de arena y grava.

Nueva localidad: Piura (Cabo Blanco), 14,5 m de profundidad (sólo conchilla).

Material examinado: 1 lote, 1 ejemplar (LaBSIM). Colector: K. Altamirano; Fecha: 19.04.2007

\section{Agradecimientos}

Los autores agradecen al Instituto del Mar del Perú (IMARPE) por haber posibilitado la participación de la Bióloga K. Altamirano en la Expedición a la costa norte del país, dentro del Proyecto "Caballito de mar", a cargo de la Bióloga Marina Quiñe. Igualmente se agradece al Consejo Superior de Investigaciones de la Universidad Nacional Mayor de San Marcos por el apoyo económico a nuestros proyectos de investigación sobre diversidad de moluscos marinos.

\section{Literatura citada}

Abbott R. T. 1974. American Seashells:The marine Mollusca of the Atlantic and Pacific Coast of North America. Van Nostrand Reinholt Co. New York. 663 pp

Alamo V. \& V. Valdivieso. 1987. Lista sistemática de moluscos marinos del Perú. Bol. Inst. Mar. Perú. Vol. extraordinario. 205 pp., 406 figs.

Dall W. H. 1909. Report on a collection of shells from Peru, with a summary of the littoral marine mollusca of the Peruvian Zoological Province. Proc. U.S.N. Mus. 37(1704): 147294.

DuShane H. 1989. The range for Macron aethiops (Reeve, 1847). The Festivus 21(7): 60-61, figs. 1-2.

DuShane H. \& E. Brennan. 1969. A preliminary survey of mollusks for Consag Rock and adjacent areas, Gula of California, México. The Veliger 11(4):351-363.

Gray, J. E. 1857. Guide to the Systematic Distribution of Mollusca in the British Museum. Part I, London.

Hendrickx M. \& A. Toledano.1994. Catálogo de Moluscos Pelecypodos, Gasterópodos y Poliplacóforos. Catálogo de Referencia, Estación Mazatlán, ICML; UNAM. Inst. Cien. del Mar y Limnología, UNAM.. 71 pp.

Keen A.M. 1971. Sea shells of tropical west America. 2th ed. Stanford Univ. Press, California. 1064 pp.

Mulliner M. 1996. Dredging around Isla Danzante, Gulf of California, México or what you find at the end of a line. The Festivus 28(6): 62-70, figs. 1-17

Paredes C. \& F. Cardoso. 1998. Nuevos registros de gasterópodos para el litoral peruano. Rev. peru. biol.. 5 (2): 118-122

Paredes C., P. Huaman, F. Cardoso, R. Vivar \& V. Vera. 1999. Estado actual del conocimiento de los moluscos acuáticos en el Perú. Rev. peru. biol. 6(1): 5-47

Paredes C. \& F. Cardoso. 2001a. Nuevos registros de Protobranchia (Mollusca: Bivalvia) para el mar peruano. Rev. per. Biol. 8(1): $5-10$

Paredes C. \& F. Cardoso. 2001b. El género Donax en la Costa Peruana (Bivalvia: Tellinoidea). Rev. per. Biol. 8(2): 83-93

Paredes C. \& F. Cardoso. 2003. Adiciones a los moluscos bivalvos marinos del Perú. Rev. per. biol. 10(1): 53-58

Paredes C. \& F. Cardoso. 2007. La Familia Calyptraeidae en el Perú. Rev. perú. biol. 13 (3): 177-184

Paredes C. \& F. Cardoso. 2008. Nuevos registros de bivalvos marinos para el Perú. Rev. per. biol. 15(1): 11-14 
Paredes et Al.

Paredes C., A. Indacochea, F. Cardoso \& K. Ortega. 2005. Familia Ellobiidae (Gastropoda: Archaeopulmonata) en el litoral peruano. Rev. peru. biol. 12(1): 69-76.

Ramírez R., C. Paredes \& J. Arenas. 2003. Moluscos del Perú. Rev. Biol. Trop. (Suppl. 3): 225-284

Rodriguez L. 2008. Impact of a native predatory whelk on cultivated oysters (Crassostrea gigas) crops in San Quintin Bay, Mexico. Aquaculture Research. 2008: 1-9. doi:10.1111/ j.1365-2109.2008.02110.x

Skoglund C. 2002. Panamic Province Molluscan Literature: Additions and Changes From 1971 through 2001. The Festivus 33 (Supplement): 286 pp. 